ISSN 1112-9867

http://www.jfas.info

\title{
DESORPTION OF Te CAPPING LAYER FROM ZnTe (100): AUGER SPECTROSCOPY, LOW-ENERGY ELECTRON DIFFRACTION AND SCANNING TUNNELING MICROSCOPY
}

K. K. Sossoe ${ }^{1,3}$, M. M. Dzagli ${ }^{1,}$,, A. Sylla ${ }^{2}$, B. Grandidier ${ }^{3,}$ M. A. Mohou ${ }^{1}$, T. J. Zoueu ${ }^{4}$, S. Toure $^{2}$

${ }^{1}$ Laboratoire de Physique des Composants à Semi-conducteurs, Université de Lomé, Togo. ${ }^{2}$ Laboratoire d'Energie Solaire, Université Félix Houphouët BOIGNY, Abidjan, Côte d'Ivoire

${ }^{3}$ Institut d'Electronique et de Microélectronique et de Nanotechnologies (IEMN), CNRS, UMR 8520, Département ISEN, 41 bd Vauban, 59046 Lille Cedex, France

${ }^{4}$ DFR-GEE, Institut National Polytechnique Félix Houphouët-Boigny (INP-HB), Yamoussoukro, Cote d'Ivoire

Received: 30 October 2015 / Accepted: 20 December 2015 / Published online: 01 January 2016

\begin{abstract}
The influence of the annealing temperature to desorb a protective Te capping layer of the zinc telluride (ZnTe (100)) surface was investigated. The surface reconstruction of the ZnTe (100) upon the removal of a Te capping layer grown by the molecular beam epitaxy was characterized by different methods. Auger spectroscopy brought out the chemical composition of the surface before and after annealing; the Low-energy electron diffraction (LEED) gave information about the crystallographic structure. The surface crystallographic configurations of tellurium Te $(\mathrm{c}(2 \mathrm{x} 2))$ and $\mathrm{Te}(\mathrm{c}(2 \mathrm{x} 1))$ are confirmed by scanning tunneling microscopy (STM). Such a study reveals a phase transition from a rich-Te to a poor-Te surface as the annealing temperature increases.
\end{abstract}

Keywords: Zinc Tellure; solar cells; structural properties; optoelectronics; semiconductors.

Author Correspondence, e-mail: mdzagli@gmail.com

doi: http://dx.doi.org/10.4314/jfas.v8i1.5 


\section{INTRODUCTION}

Les semi-conducteurs II-VI sont des matériaux qui promettent énormément du point de vue des applications optoélectroniques [1], précisément dans la réalisation des dispositifs émetteurs de lumière et des détecteurs de lumière [2,3]. Principalement, issu de la famille des semi-conducteurs à gap direct avec une largeur de bande interdite de 2,26 eV (549 nm) à $300 \mathrm{~K}$ et un paramètre de maille $\mathrm{a}=6,10132 \AA$, le ZnTe est considéré comme la structure appropriée pour la réalisation de diodes émettrices de lumière verte, des photodétecteurs UV-verts et des cellules solaires multi-jonctions $[4,5,6,7,8,9,10]$. Cependant, la réalisation technique de ces dispositifs exige des couches actives de grande qualité cristalline selon le processus d'élaboration qui est généralement la croissance par épitaxie à jets moléculaires (Molecular Beam Epitaxy, MBE). Pour analyser la qualité de ces couches après croissance, il est intéressant de pouvoir les protéger avant leur transfert vers les systèmes d'analyse via un bref passage dans l'air. Une méthode de choix consiste à recouvrir l'échantillon de ZnTe par une couche amorphe de tellure et à désorber cette couche une fois le transfert effectué pour retrouver la surface originelle. Cependant la désorption thermique du tellure n'est pas bien connue et seules des surfaces de CdTe ont été étudiées pour déterminer l'énergie d'activation de désorption des éléments $\mathrm{Cd}$ et Te. Dans ce cas, la désorption du tellure se produit entre $220^{\circ} \mathrm{C}$ et $340^{\circ} \mathrm{C}[11,12]$. Dans ce travail, nous nous intéressons à la désorption d'une couche amorphe de tellure utilisée pour protéger la surface ZnTe (100). En combinant les techniques de spectroscopies AUGER avec celles de la réflexion d'électrons lents appuyées ensuite par de l'imagerie STM, nos analyses montrent l'influence de la température sur la configuration cristallographique des atomes de Te ou de $\mathrm{Zn}$ en surface suite à la désorption de la couche protectrice de tellure.

\section{MATERIEL ET METHODES}

\section{a. L'échantillon}

L'échantillon de ZnTe analysé en combinant les différentes techniques d'exploration des surfaces a été fabriqué par épitaxie par jets moléculaires (Molecular Beam Epitaxy, MBE), comme cela est décrit dans la référence [13]. Cet échantillon est formé de deux couches de 
ZnTe de $200 \mathrm{~nm}$ et $20 \mathrm{~nm}$, déposées sur un substrat de ZnTe. Une couche protectrice de tellure recouvre la surface de l'échantillon comme on l'observe sur la figure 1. Cette couche est désorbée dans une gamme de température comprise entre $250^{\circ} \mathrm{C}$ et $270^{\circ} \mathrm{C}$. Cette gamme de température se trouve dans la fourchette basse des températures généralement utilisées pour la désorption du tellure [11], ceci afin d'éviter de détériorer d'éventuelles hétérostructures II-VI qui pourraient être protégées par la suite avec cette technique, en particulier des boîtes quantiques de CdTe fabriquées sur ZnTe.

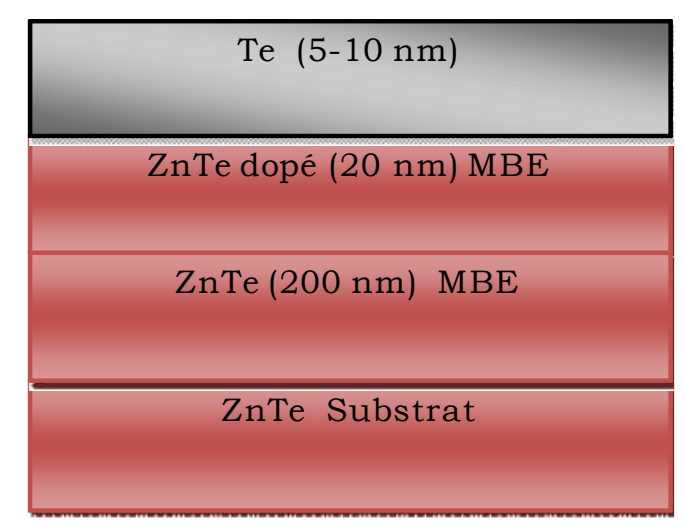

Fig. 1. Schéma de l'échantillon de ZnTe

\section{b. Techniques d'analyse de surface}

Les analyses LEED (Low Energy Electron Diffraction), la spectroscopie AUGER ont été réalisées sur la plateforme expérimentale STM du laboratoire d'IEMN-CNRS (Lille). Cette plateforme est un bâti qui abrite à la fois la tête d'un microscope à effet tunnel fonctionnant à température ambiante, l'analyseur AUGER et le LEED. Ces analyses exhibent les différentes reconstructions surfaciques apparues en fonction de la température et du temps de chauffage. L'échantillon analysé dans la chambre d'analyse sous UHV (Ultra High Vacuum) à $2,3 \times 10^{-10}$ Torr fut successivement chauffé à $250^{\circ} \mathrm{C}$ pendant $1 \mathrm{~min}, 270^{\circ} \mathrm{C}$ pendant $1 \mathrm{~min}$ et à $270^{\circ} \mathrm{C}$ pendant $1 \mathrm{~h}$.

Les images STM furent réalisées sur la plateforme STM de l'ISEN (Institut Supérieur de l'Electronique et du Numérique de Lille), avec un microscope à effet tunnel fonctionnant à basse température (5K grâce à l'Hélium liquide et $77 \mathrm{~K}$ pour l'azote liquide). Un traitement numérique d'images est réalisé pour améliorer la résolution des images STM grâce au logiciel 
de traitement d'images WsXM.

\section{RESULTATS ET DISCUSSION}

Avant désorption de la couche de tellure protectrice, l'échantillon de ZnTe est soumis à un faisceau d'électrons énergétiques afin de produire des électrons Auger pour déterminer la nature chimique des atomes de surface. Le spectre obtenu (Fig. 2a) présente un premier pic à basse énergie $(284,6 \mathrm{eV})$ typique de l'atome de carbone, puis une succession de pics, dont le plus intense est situé à $483 \mathrm{eV}$, caractéristique de l'atome de tellure [14,15]. Dans la gamme $850 \mathrm{eV}-1050 \mathrm{eV}$ aucun pic n'est visible.

La présence d'atomes de carbone sur la surface s'expliquerait par d'éventuelles contaminations durant le processus de croissance ou l'adsorbtion de molécules organiques à la surface de l'échantillon lors de son bref transfert dans l'air. En effet, le pic associé au carbone disparaît en chauffant $1^{\prime}$ 'échantillon à $250^{\circ} \mathrm{C}$ pendant $1 \mathrm{~min}$, comme le montre la nouvelle analyse Auger (Fig. 2a). Le pic de tellure est lui toujours présent, mais cette fois-ci est associé à une série de pic dans la gamme $850 \mathrm{eV}-1050 \mathrm{eV}$, signature de la présence de l'élement zinc à la surface de l'échantillon (Fig. 2b).
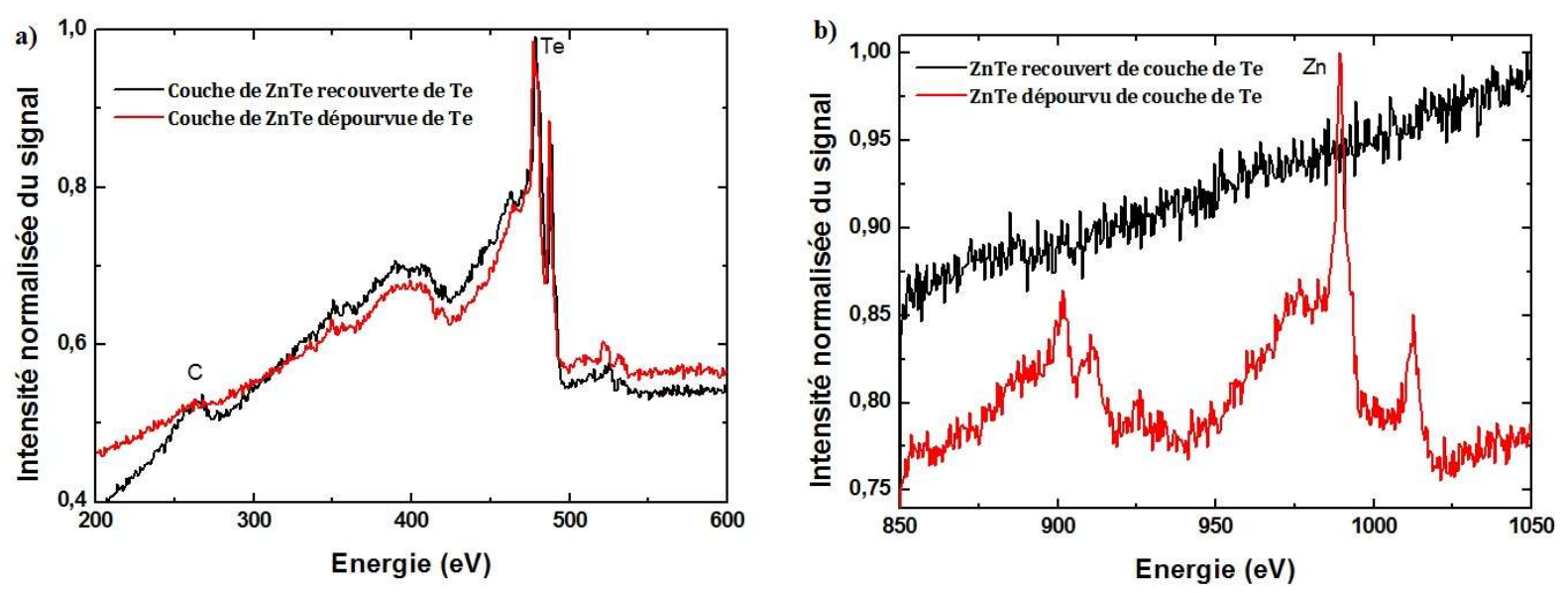

Fig. 2. Spectres Auger d'un échantillon de ZnTe avant et après désorption de la couche protectrice de Te sur la gamme : a)-(200-800) eV et b)-(850-1050) eV.

Ces analyses révèlent ainsi la désorption de la couche protectrice de tellure. Un chauffage à $270^{\circ} \mathrm{C}$ pendant une minute n'apporte aucun changement. La figure 3 est une image obtenue par microscopie à effet tunnel après chauffage. Cette image montre trois terrasses atomiques 
sur lesquelles de fines rangées sont devinées. Ces rangées correspondent aux rangées atomiques en surface de l'échantillon ZnTe (100). Ces variations sont plus visibles sur le profil. Entre 0 et $20 \mathrm{~nm}$, un premier plan est visible. Un deuxième plan lui succède entre 20 et $80 \mathrm{~nm}$, puis un troisième entre 80 et $100 \mathrm{~nm}$.

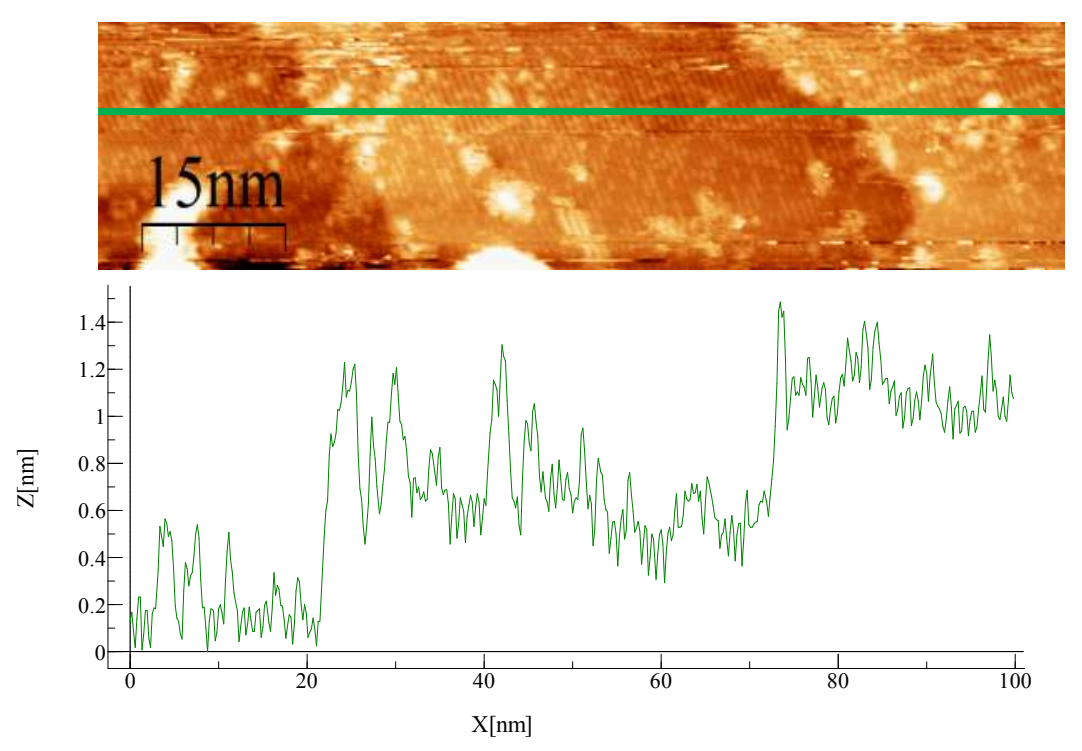

Fig. 3. Image $\mathrm{STM}\left(\mathrm{V}_{\mathrm{gap}}=-3,2 \mathrm{~V} ; \mathrm{I}=2 \mathrm{pA}\right)$ montrant les strates atomiques (en haut) et le profil des plans (en bas).

Après un chauffage prolongé pendant $1 \mathrm{~h}$ à température constante de $270^{\circ} \mathrm{C}$, un nouvel examen sous l'analyseur fut réalisé. Les spectres obtenus suivant les deux gammes d'énergies des électrons comme précédemment présente :

$\checkmark$ suivant la gamme $200 \mathrm{eV}-600 \mathrm{eV}$; deux pics caractéristiques (fig.4.a) d'atomes de carbone et tellure,

$\checkmark$ suivant la gamme $850 \mathrm{eV}-1050 \mathrm{eV}$, un pic caractéristique (fig.4.b) de l'atome de zinc.

La présence de traces de carbone indique une diffusion d'atomes de carbone vers la surface. Ces atomes pourraient provenir d'une contamination pendant la croissance de la couche de ZnTe. L'intensité des pics associés à l'élément zinc ne varie pas. Par contre, les pics associés au tellure semblent un peu moins intense, ce qui indiquerait une différence de la composition de la surface et donc un changement de la stoïchiométrie de la surface. 

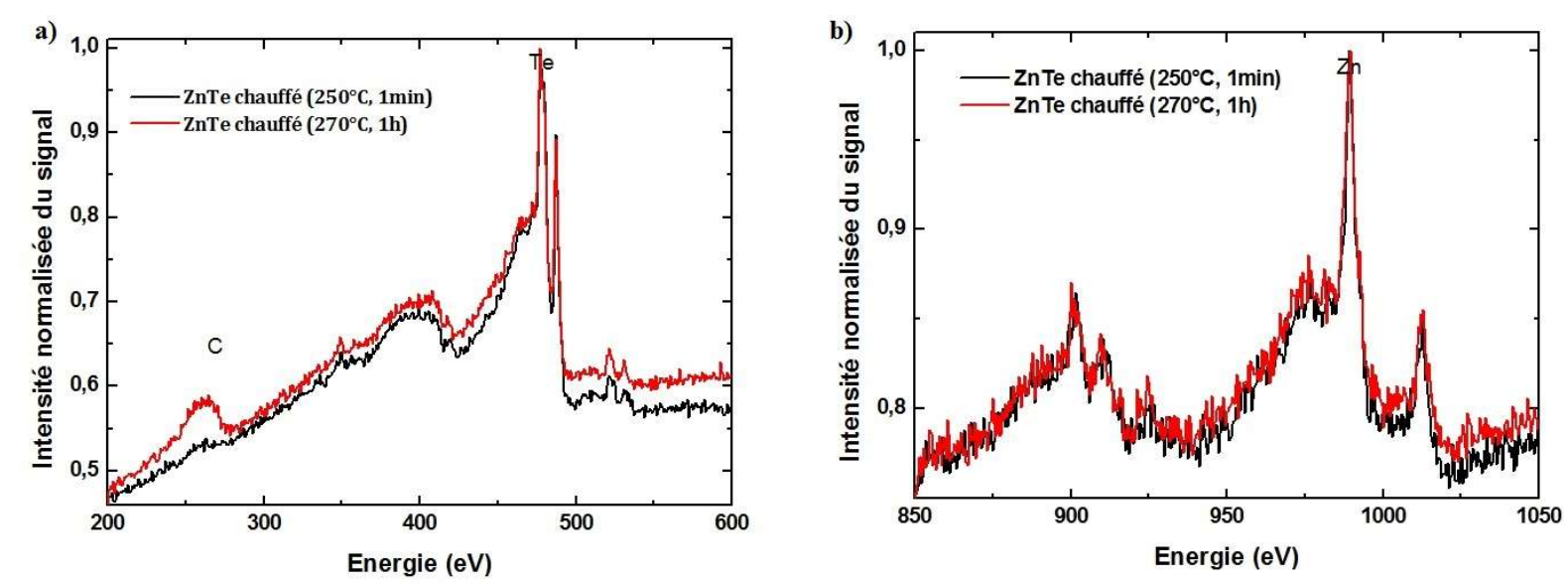

Fig. 4. Spectres Auger après chauffage à $\left(250^{\circ} \mathrm{C}, 1 \mathrm{~min}\right)$ et $\left(270^{\circ} \mathrm{C}, 1 \mathrm{~h}\right)$ sur les gammes d'énergie : a)-(200-600) eV et b)-(850-1050) eV

Afin de retrouver la disposition cristallographique des atomes en surfaces, nous avons établi, parallèlement aux analyses Auger, un examen de la surface par diffraction d'électrons lents (LEED). Cette étude a été effectuée après chaque chauffage; $250^{\circ} \mathrm{C}, 1 \mathrm{~min} / 270^{\circ} \mathrm{C}, 1 \mathrm{~min} /$ $270^{\circ} \mathrm{C}, 1 \mathrm{~h}$. Les clichés des figures de diffraction sont présentés sur la figure 5 .

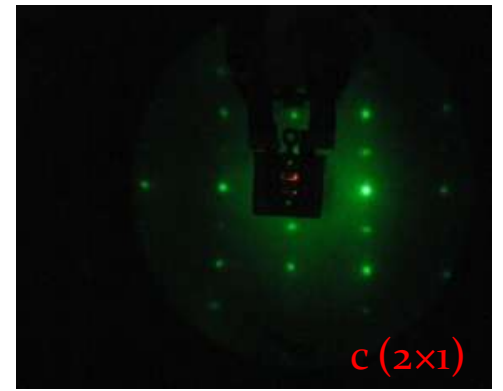

$\left(250^{\circ} \mathrm{C}, 1 \mathrm{~min}\right)$

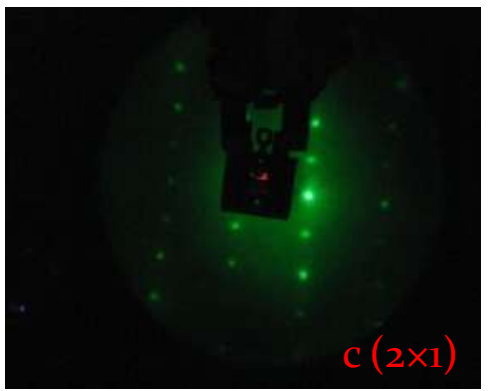

$\left(270^{\circ} \mathrm{C}, 1 \mathrm{~min}\right)$

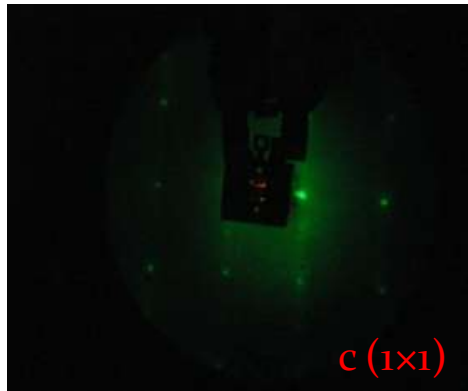

$\left(270^{\circ} \mathrm{C}, 1 \mathrm{~h}\right)$

Fig. 5. Figures de diffraction obtenues par le LEED

Il ressort des figures de diffraction deux principales configurations cristallographiques surfaciques. En effet, après un chauffage de $250^{\circ} \mathrm{C}$ pendant 1 min puis un autre à $270^{\circ} \mathrm{C}$ pendant $1 \mathrm{~min}$ qui a permis la désorption de la couche protectrice de tellure, l'analyse au LEED révèle une reconstruction surfacique des atomes de tellure de type $c(2 \times 1)$. Ce type d'arrangement suppose des rangées atomiques privilégiées dans un sens. Lorsqu'on soumet 
notre échantillon de ZnTe à un chauffage de $270^{\circ} \mathrm{C}$ pendant $1 \mathrm{~h}$ et qu'on réalise une nouvelle analyse LEED, la figure de diffraction montre une nouvelle configuration cristallographique en surface des atomes de tellure, de type $\mathrm{c}(1 \mathrm{x} 1)$, qui serait causée par une désorption prononcée d'atomes de tellure.

Des études réalisées sur la croissance du ZnTe (100) par MBE par Tatarenko et al. [16] montrent qu'au cours de la croissance, la surface de ZnTe se reconstruit suivant la maille de type $\mathrm{c}(2 \mathrm{x} 2)$ sous flux permanent de $\mathrm{Zn}$ autour de $380^{\circ} \mathrm{C}$ et se transforme en $\mathrm{c}(2 \mathrm{x} 1)$ si on arrête le flux. Ce groupe a proposé des modèles atomiques des mailles cristallographiques surfaciques Te $(c(2 \times 2)$ et $c(2 \times 1))$ (fig.6). De même sous flux permanent de Te entre $225^{\circ}$ et $260^{\circ} \mathrm{C}$, la couche de tellure sur la surface exhibe une reconstruction suivant la maille $\mathrm{c}(2 \times 2)$. Si on arrête de même le flux de Te, c'est-à-dire une surface pauvre en tellure, une nouvelle reconstruction de maille $\mathrm{c}(2 \times 1)$ apparaît et demeure au-delà de $260^{\circ} \mathrm{C}[17,18]$. Ils ont aussi remarqué un changement de structure qui n'est plus le c $(2 \times 1)$ lorsqu'on chauffe vers les $380^{\circ}$ sans pour autant préciser exactement le type de reconstruction observée.

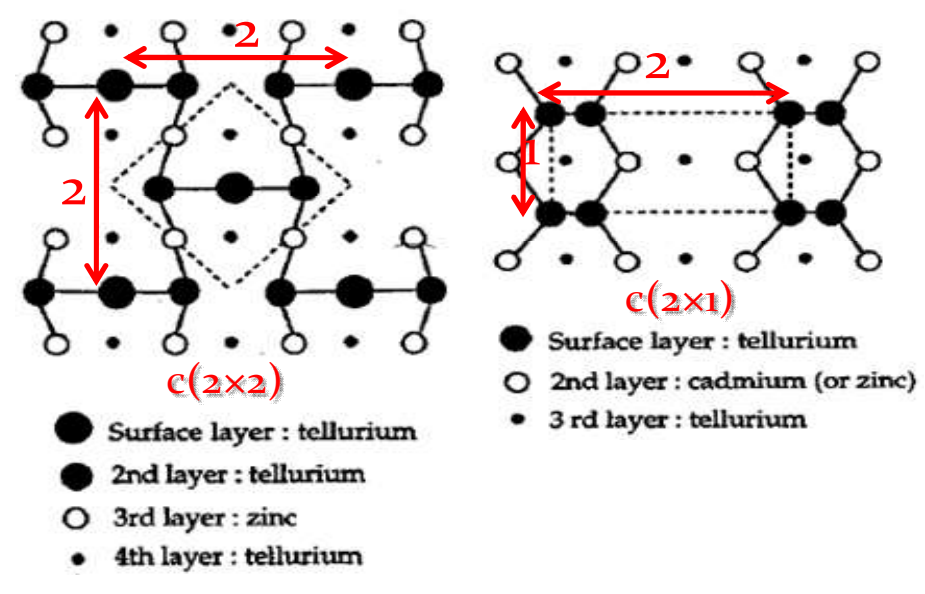

Fig. 6. Modèles atomiques des mailles cristallographiques surfaciques Te $(\mathrm{c}(2 \times 2))$ et $\operatorname{Te}(\mathrm{c}(2 \mathrm{x} 1))[16]$.

Des analyses complémentaires réalisées par microscopie à effet tunnel à basse température après le premier chauffage confirment les observations faites au LEED. Les images obtenues mettent en exergue la reconstruction de la surface ZnTe (100). L'observation de rangées atomiques indique une reconstruction $\mathrm{c}(2 \mathrm{x} 1)$ et la présence de protrusions claires sur les rangées donnent à certains endroits la structure $\mathrm{c}(2 \times 2)$, comme le montre la figure 7 . Ces 
protrusions peuvent donc être associées à des atomes de tellure.

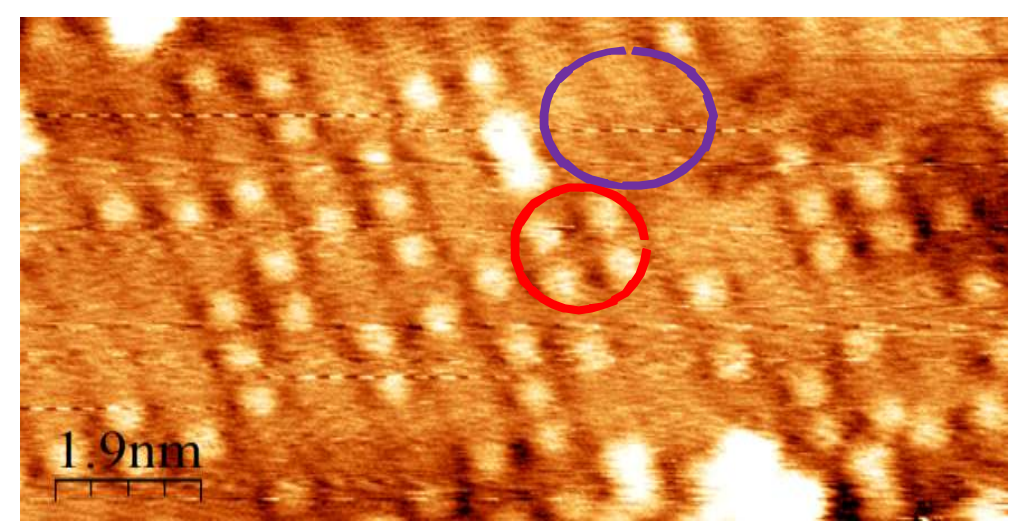

Fig. 7. Image STM à haute résolution atomique de la surface ZnTe (100) avec une pointe en tungstène obtenue sous la condition $V=-2,7 \mathrm{~V} ; \mathrm{I}=10 \mathrm{pA}$. La zone encerclée en rouge présente une reconstruction $\mathrm{c}(2 \times 2)$ et en violet une reconstruction $\mathrm{c}(2 \times 1)$ qui prédomine sur l'image en arrière-plan sur la couche inférieure.

L'exploitation des images STM nous a permis d'évaluer la distance entre deux rangées atomiques dans la configuration $\mathrm{c}(2 \mathrm{x} 1)$. La valeur de cette distance est donnée par la relation $\frac{a \sqrt{2}}{2}=4,314 \stackrel{\circ}{A}$ pour un paramètre de maille $a=6,10132 \stackrel{\circ}{A}$.

Nous n'avons pas pu obtenir des images montrant la structure $\mathrm{c}(1 \mathrm{x} 1)$ du fait que l'apex de la pointe dans les conditions de notre expérimentation n'était pas capable de distinguer des détails de l'ordre du paramètre de maille. Kanazawa et al. ont aussi remarqué que les couches atomiques superficielles sur le ZnTe (110) sont des surfaces reconstruites [19].

\section{CONCLUSION}

Dans ce travail nous avons utilisé différentes techniques de caractérisations de surfaces semi-conductrices pour étudier la désorption d'une couche protectrice de tellure à partir d'un échantillon ZnTe (100). Cette désorption thermique conduit à une reconstruction de surface qui varie en fonction de la stoïchiométrie des atomes de tellure : (1) pour des températures inférieures à $250^{\circ} \mathrm{C}$ on remarque une reconstruction $\mathrm{c}(2 \times 2)$ suite à une trimérisation des 
atomes de tellure; (2) entre $250^{\circ} \mathrm{C}-270^{\circ} \mathrm{C}$ on observe une reconstruction $\mathrm{c}(2 \times 1)$ due à une désorption d'atomes de tellure et une dimérisation des atomes recouvrant la surface ; (3) un chauffage au-delà de $270^{\circ} \mathrm{C}$ et sur une longue durée provoque un changement de phase avec l'apparition d'une nouvelle reconstruction de type $c(1 \times 1)$. Ce travail montre clairement que l'évaporation d'une couche amorphe de tellure sur des matériaux semi-conducteurs II-VI permet de récupérer une surface cristalline, dont la structure est directement liée à la concentration des atomes de tellure.

\section{REMERCIEMENTS}

Les auteurs remercient Maxime Berthe, Jean-Philippe Nys, Yannick Lambert, Pierre Capiod, pour leur apport technique et leurs discussions fructueuses. Ils remercient également l'institut Néel à Grenoble et en particulier Dr. H. Boukari pour la croissance des échantillons. Nos gratitudes sont adressées à l'AUF (Agence Universitaire de la Francophonie) pour leur soutien financier (Convention «BAO-2012-05-U-52220FT2E0») et aux universités partenaires pour leur soutien divers.

\section{REFERENCES}

[1] Bozzini B, Baker M A, Cavallotti P L, et al. Thin Solid Films, 2000, 361, 388-395, doi:10.1016/S0040-6090(99)00808-1.

[2] Marfaing Y. Journal of crystal growth, 1996, 161 (1), 205-213, doi:10.1016/0022-0248(95)00641-9.

[3] Fan Y, Han J, He L, et al. Applied physics letters, 1992, 61 (26), 3160-3162, http://dx.doi.org/10.1063/1.107945.

[4] Wang S, Ding D, Liu X, et al. Journal of Crystal Growth, 2009, 311 (7), 2116-2119, doi:10.1016/j.jcrysgro.2008.09.189.

[5] Ding D, Wu S-N, Wang S, et al. In : IEEE Lasers and Electro-Optics Society, 2008. LEOS 2008. 21st Annual Meeting of the. IEEE, 2008, 93-94, 10.1109/LEOS.2008.4688504.

[6] Zhang Y-H, Yu S-Q, Johnson S R, et al. In: Proceedings of the 33rd IEEE Photovoltaic Energy Specialist Conference, 2008, 20-25, 10.1109/PVSC.2008.4922555 
[7] Song K W, Costi R, et Bulović V. Advanced Materials, 2013, 25 (10), 1420-1423, $\underline{10.1002 / \mathrm{adma} .201203079 .}$

[8] Jun Y-W, Choi C-S, Cheon J. Chem Commun (1), 2001, 101-102, 10.1039/B008376N.

[9] Davami K, Lee J-S, et Meyyappan M. Transactions on Electrical and Electronic Materials, 2011, vol. 12 (6), 227-233, 10.4313/TEEM.2011.12.6.227.

[10] Chuang C-H M, Brown P R, Bulović V, et al. Nature materials, 2014,. 13 (8), 796-801, doi: $10.1038 /$ nmat3984

[11] Tatarenko S, Daudin B, Brun D, et al. Physical Review B, 1994, 50 (24), 18479, http://dx.doi.org/10.1103/PhysRevB.50.18479

[12] Wu Y S, Becker C R, Waag A, et al. Applied physics letters, 1993, 62 (13), 1510-1512, $\underline{\mathrm{http}: / / \mathrm{dx} . \text { doi.org/10.1063/1.108623 }}$

[13] C. Le Gall, A. Brunetti, H. Boukari, L. Besombes, Phys. Rev. Lett., 2011,107 (5), 057401. http://dx.doi.org/10.1103/PhysRevLett.107.057401

[14] F. Wang, A. Schwartzman, A. L. Farhenbruch, R. Sinclair, R. H. Bube and C M. Stahle, J. Appl. Phys., 1987, 62 (4), 1469. http://dx.doi.org/10.1063/1.339653

[15] P. Loaeza, O. Solorza, J. Mater. Sci. Lett. 1990, 9(1), 11-13. 10.1007/BF00722853

[16] Tatarenko S, Daudin B, et Brun-Le Cunff D. Applied physics letters, 1995, 66 (14), 1773-1775, http://dx.doi.org/10.1063/1.113363

[17] Daudin B, Brun-Le Cunff D, et Tatarenko S. Surface science, 1996, 352, 99-104. doi:10.1016/0039-6028(95)01103-X

[18] Daudin B, Tatarenko S, et Brun-Le Cunff D. Physical Review B, 1995, 52, (11), 7822. http://dx.doi.org/10.1103/PhysRevB.52.7822

[19] Kanazawa K, Yoshida S, Shigekawa H, et al. Science and Technology of Advanced Materials, 2015, 16 (1), 015002. http://dx.doi.org/10.1088/1468-6996/16/1/015002

\section{How to cite this article:}

Sossoe K. K., Dzagli M. M., Sylla A, Mohou M A, Zoueu T J, Grandidier B, Toure S. Desorption of Te capping layer from $\mathrm{ZnTe}(100)$ : Auger spectroscopy, low-energy electron diffraction and scanning tunneling microscopy. J. Fundam. Appl. Sci., 2016, 8(1), 73-82. 